\title{
miR-125b is associated with renal cell carcinoma cell migration, invasion and apoptosis
}

\author{
LU JIN ${ }^{1-3}$, ZENG ZHANG ${ }^{1-3}$, YIFAN LI ${ }^{1-3}$, TAO HE ${ }^{1,3,4}$, JIA HU $^{1,3,4}$, JIAJU LIU $^{1,3,5}$, \\ MINGWEI CHEN ${ }^{2,3}$, YAOTING GUI ${ }^{3}$, YUN CHEN ${ }^{6}$ and YONGQING LAI ${ }^{1,3}$
}

\begin{abstract}
${ }^{1}$ Department of Urology, Peking University Shenzhen Hospital, Shenzhen, Guangdong 518036; ${ }^{2}$ Department of Urology, Anhui Medical University, Hefei, Anhui 230032; ${ }^{3}$ The Guangdong and Shenzhen Key Laboratory of Male Reproductive Medicine and Genetics, Shenzhen, Guangdong 518036; ${ }^{4}$ Department of Urology, Guangzhou Medical University, Guangzhou, Guangdong 511436; ${ }^{5}$ Department of Urology, Shantou University Medical College, Shantou, Guangdong 515041;

${ }^{6}$ Department of Ultrasound, Peking University Shenzhen Hospital, Shenzhen, Guangdong 518036, P.R. China
\end{abstract}

Received January 25, 2016; Accepted December 16, 2016

DOI: $10.3892 / 01.2017 .5985$

\begin{abstract}
MicroRNA (miR)-125b has been identified as deregulated in a number of types of cancer. Previous studies have detected the expression of miR-125b in clear cell renal cell carcinoma (ccRCC) tissues by in situ hybridization and revealed that miR-125b was upregulated in ccRCC tissues, and was associated with recurrence and survival of patients with ccRCC. However, the function of miR-125b in RCC remains unclear. Thus, the expression of miR-125b was detected with quantitative polymerase chain reaction (qPCR) in 24 paired $\mathrm{RCC}$ and adjacent normal tissues. The result of qPCR showed that miR-125b was upregulated in RCC tissues. Furthermore, the function of miR-125b in RCC (786-O and ACHN) cells was detected by transfecting miR-125 mimic or inhibitor to upregulate or downregulate miR-125b expression. Cell proliferation assays (MTT and Cell Counting Kit-8), cell mobility assays (cell scratch and Transwell assay) and a cell apoptotic assay (flow cytometry assay) were performed to assess the function of miR-125b on RCC cells. Results from the assays demonstrated that overexpression of miR- $125 \mathrm{~b}$ could promote cell migration and invasion, and reduce the cell apoptotic rate. It was also revealed that downregulation of miR-125b could reduce cell migration and invasion, and induce cell apoptosis. However, the results of the cell proliferation assay revealed that miR-125b had no significant effect on cell proliferation. Not only could miR-125b predict recurrence and survival of
\end{abstract}

Correspondence to: Professor Yongqing Lai, Department of Urology, Peking University Shenzhen Hospital, 1120 Lianhua Road, Shenzhen, Guangdong 518036, P.R. China

E-mail: yqlord@163.com

Professor Yun Chen, Department of Ultrasound, Peking University Shenzhen Hospital, 1120 Lianhua Road, Shenzhen, Guangdong 518036, P.R. China

E-mail: cyun126@126.com

Key words: microRNA, miR-125b, renal cell carcinoma, oncogene
ccRCC; the present study revealed that miR-125b could regulate RCC cell migration, invasion and apoptosis. Additional studies are required to determine the mechanism of miR-125b in RCC cells and define the target genes of miR-125b in RCC.

\section{Introduction}

Renal cell carcinoma ( $\mathrm{RCC}$ ) is the most common type of renal cancer, accounting for $\sim 3 \%$ of malignancies in adults (1). The prognosis of advanced cancer is reported to be poor, with a 5 -year survival rate of 5-10\% (2,3). RCC has no characteristic symptom, so $\sim 30 \%$ of patients are presented with metastatic disease at the time of diagnosis. Radical nephrectomy is the primary treatment choice for patients with RCC, due to the resistance of radiotherapy and chemotherapy $(4,5)$. However, $20-40 \%$ of patients developed recurrence following the curative nephrectomy (6). Thus, it is important to find a biomarker of RCC for early diagnosis and targeted therapy based on the mechanisms of RCC tumorigenesis.

MicroRNAs (miRNAs, miRs) are a class of small non-coding RNA that regulates gene expression by targeting the 3' untranslated region (UTR) of targeted mRNAs (7-9). Previous studies indicated that the abnormal expression of miRNA was involved in tumorigenesis with a tissue-specific expression pattern $(6,7,10)$. miRNAs have been revealed to be a potential choice of biomarker for a number of diseases, particularly tumors (10-12). For example, miR-210 has been described as a potential biomarker for clear cell RCC (ccRCC) (11). In addition, miRNAs may also regulate cellular processes including proliferation, apoptosis and differentiation (10). Therefore, it is essential to identify the role of miRNAs in tumorigenesis.

Studies investigating miR-125b (also termed miR-125b-5p) demonstrated that miR-125b was deregulated in numerous types of tumor, including osteosarcoma (12), hepatocellular carcinoma (13) and breast cancer (14). The function of miR-125b in a number of cancer subtypes, including osteosarcoma and gastric cancer, was explored $(15,16)$. In osteosarcoma, miR-125b was described as a tumor suppressor (15), and in gastric cancer, miR-125b functions as an oncogene (16). 
Fu et al (17) detected the expression of miR-125b in ccRCC tissues by in situ hybridization (ISH) and revealed that miR-125b was upregulated in ccRCC tissues, and the expression level of miR-125b is associated with the recurrence and survival of patients with ccRCC following nephrectomy. However, the function and mechanism of miR-125b in RCC remain unclear. In the present study, quantitative polymerase chain reaction (qPCR) was performed to detect the expression of miR-125b in RCC tissues and paired adjacent normal tissues. The function of miR-125b in RCC cell proliferation, migration, invasion and apoptosis was explored.

\section{Materials and methods}

Sample collection. In total, 24 paired RCC and adjacent normal tissues were collected from Peking University Shenzhen Hospital (Shenzhen, China) between December 2012 and December 2014. Collection and the use of the tissues were reviewed and approved by the Ethics Committees of Peking University Shenzhen Hospital, and written informed consent was obtained from all the patients. The tissues were immersed in RNAlater (Qiagen GmbH, Hilden, Germany) for $30 \mathrm{~min}$, and were then stored at $-80^{\circ} \mathrm{C}$ for further study. The adjacent normal tissues were $2 \mathrm{~cm}$ away from the visible RCC lesions. Following collection, the tissues were reviewed and classified by hematoxylin and eosin staining (Beyotime Institute of Biotechnology, Shanghai, China). The clinical and pathological characteristics of the patients are presented in Table I.

Cell culture and transfection. Human embryo kidney 293T cells (Type Culture Collection of the Chinese Academy of Medical Sciences, Shanghai, China), RCC 786-O and ACHN cells (both American Type Culture Collection, Manassas, VA, USA) were used in the present study. Cells were cultured in Dulbecco's modified Eagle's medium (DMEM) (Gibco; Thermo Fisher Scientific. Inc., Waltham, MA, USA) supplemented with $10 \%$ fetal bovine serum (FBS) (Gibco; Thermo Fisher Scientific, Inc.), $1 \%$ antibiotics $(100 \mu \mathrm{l} / \mathrm{ml}$ penicillin and $100 \mathrm{mg} / \mathrm{ml}$ streptomycin sulfates) and $1 \%$ glutamine, at $37^{\circ} \mathrm{C}$ in a $5 \% \mathrm{CO}_{2}$ atmosphere. Synthesized miR-125b mimic or inhibitor (GenePharma, Shanghai, China) was transfected into cells with Lipofectamine 2000 (Invitrogen; Thermo Fisher Scientific, Inc.) mixed in Opti-MEM ${ }^{\circledR}$ I reduced serum medium (Gibco; Thermo Fisher Scientific, Inc.) for upregulation or downregulation of miR-125b. qPCR was performed to detect the expression of miR-125b following transfection. Sequences are presented in Table II.

RNA extraction, $c D N A$ synthesis and quantitative polymerase chain reaction $(q P C R)$. Total RNA was extracted from the tissues and cells following transfection with TRIzol reagent (Invitrogen; Thermo Fisher Scientific, Inc.) and purified with the RNeasy Maxi kit (Qiagen $\mathrm{GmbH}$ ), according to the manufacturer's protocol. NanoDrop 2000/2000c (Thermo Fisher Scientific, Inc.) was used to detect the concentration of extracted RNA. RNA (1 $\mu \mathrm{g})$ was used to perform reverse transcription with the miScript reverse transcription kit (Qiagen $\mathrm{GmbH}$ ) to get cDNA. The following thermocycling conditions were used: $37^{\circ} \mathrm{C}$ for $60 \mathrm{~min}$ and $95^{\circ} \mathrm{C}$ for $5 \mathrm{~min}$. qPCR was then performed with the miScript SYBR ${ }^{\circledR}$ Green PCR kit (Qiagen
Table I. Clinicopathological features of RCC patients.

\begin{tabular}{lc}
\hline Characteristics & Number of cases \\
\hline Mean age range, years & $50(25-70)$ \\
Gender & 15 \\
Male & 9 \\
Female & \\
Histological type & 20 \\
Clear cell & 4 \\
Papillary & \\
pT-stage & 13 \\
T1 & 7 \\
T2 & 4 \\
T3+T4 & \\
Fuhrman grade & 10 \\
I & 10 \\
II & 4 \\
III+IV & \\
AJCC clinical stages & 12 \\
I & 7 \\
II & 5 \\
III+IV & \\
\hline
\end{tabular}

pT, primary tumor; AJCC, American Joint Committee on Cancer.

Table II. Sequences used in the present study.

\begin{tabular}{ll}
\hline Name & \multicolumn{1}{c}{ Sequence } \\
\hline $\begin{array}{l}\text { miR-125b mimic } \\
\text { Sense }\end{array}$ & 5'-UCCCUGAGACCCUAACUUG \\
& UGA-3' \\
Antisense & 5'-ACAAGUUAGGGUCUCAGGG \\
& AUU-3' \\
Negative control & \\
Sense & 5'-UUCUCCGAACGUGUCACGUTT-3' \\
Antisense & 5'-ACGUGACACGUUCGGAGAATT-3' \\
miR-125b & 5'-UCACAAGUUAGGGUCUCAG \\
inhibitor & GGA-3' \\
Inhibitor negative & 5'-CAGUACUUUUGUGUAGUA \\
control & CAA-3' \\
U6 primer & \\
Forward & 5'-CTCGCTTCGGCAGCACA-3' \\
Reverse & 5'-ACGCTTCACGAATTTGCGT-3' \\
miR-125b primer & \\
Forward & 5'-TCCCTGAGACCCTAACTTGTGA-3' \\
Reverse & Universal primer (miScript SYBR-Green \\
& PCR kit) \\
&
\end{tabular}

$\mathrm{GmbH})$ to detect the expression of miR-125b on the Roche Lightcycler 480 quantitative PCR system, following the manufacturer's protocol (Roche Diagnostics, Basel, Switzerland) 

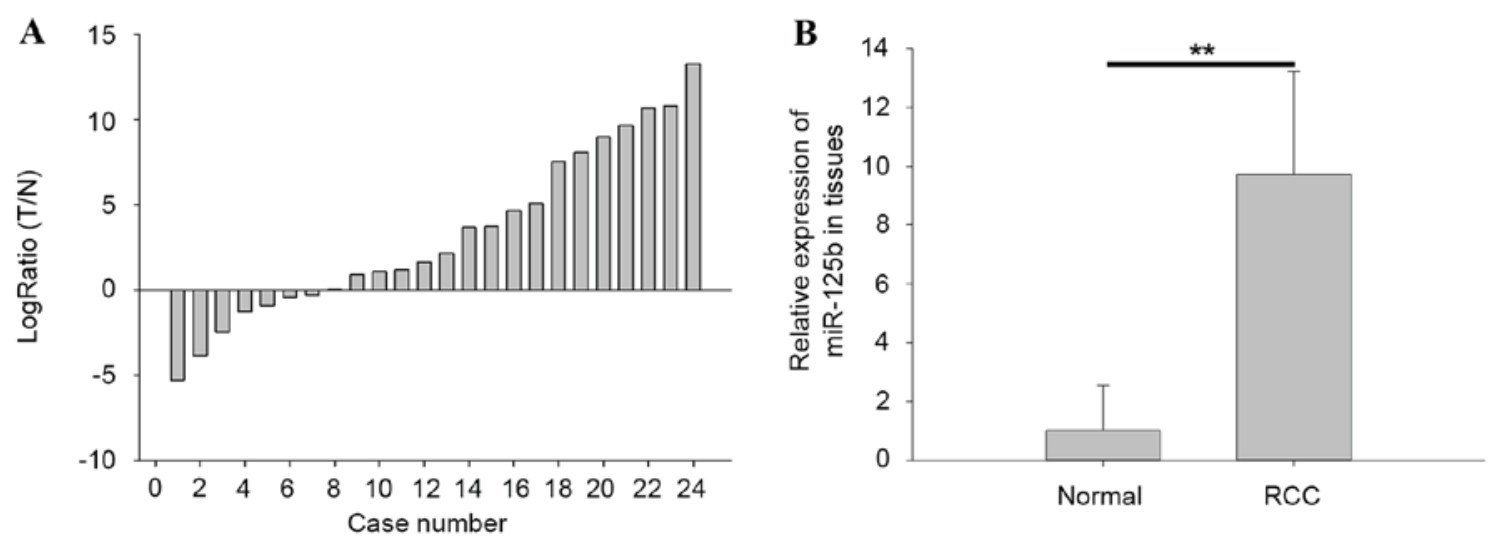

Figure 1. Expression of miR-125b in 24 paired tissues. (A) $\log _{2}$ Ratios (T/N) of miR-125b expression in 24 paired RCC tissues. (B) Relative expression level of miR-125b in RCC tissues is higher than normal tissues ( $\left.{ }^{* *} \mathrm{P}<0.01\right)$. RCC, renal cell carcinoma; miR-125b, microRNA-125b; T, tumor tissues; N, normal adjacent tissues.

and performed in triplicate. Primers used are presented in Table II. U6 was used as an internal control. Universal primer provided by the miScript SYBR Green PCR kit was used as a reverse primer of miR-125b. The following thermocycling conditions were used in the qPCR reaction: $95^{\circ} \mathrm{C}$ for $1 \mathrm{~min}$; 40 cycles of $95^{\circ} \mathrm{C}$ for $10 \mathrm{sec}, 55^{\circ} \mathrm{C}$ for $30 \mathrm{sec}$ and $70^{\circ} \mathrm{C}$ for $30 \mathrm{sec}$. The expression of miR-125b was analyzed using the $2^{-\Delta \Delta \mathrm{Cq}}$ method (18).

Cell proliferation assay. MTT and Cell Counting Kit- 8 (CCK-8) assays were performed to assess proliferative ability of 786-O and ACHN cells following transfection. In each well of a 96-well plate, $\sim 3,000$ cells were seeded and $24 \mathrm{~h}$ later transfected with 5 pmol miR-125b mimics, inhibitors, mimic negative control (NC) or inhibitor NC. For the MTT assay, $20 \mu 1$ MTT (5 mg/ml; Sigma-Aldrich; Merck Millipore, Darmstadt, Germany) was added to the wells, which were detected at $0,24,48$ and $72 \mathrm{~h}$ following transfection, and then incubated at $37^{\circ} \mathrm{C}$ for $4 \mathrm{~h}$. Subsequently, the mixed medium was replaced by $150 \mu 1$ dimethylsulfoxide (DMSO; Sigma-Aldrich; Merck Millipore) and then agitated at $0.04 \times g$ for $15 \mathrm{~min}$ at room temperature. The optical density (OD) value of each well was then evaluated using the ELISA microplate reader (Bio-Rad Laboratories, Inc., Hercules, CA, USA) at a wavelength of $490 \mathrm{~nm}$. For the CCK-8 assay, $15 \mu \mathrm{l} \mathrm{CCK-8} \mathrm{(Beyotime} \mathrm{Institute}$ of Biotechnology) was added to the wells, which were detected at $0,24,48$ and $72 \mathrm{~h}$ post-transfection. The OD value of each well was measured at a wavelength of $490 \mathrm{~nm} 1.5 \mathrm{~h}$ later. The experiments were performed in triplicate and repeated at least three times.

Cell scratch assay. The cell scratch assay was performed to assess the migratory ability of 786-O and ACHN cells following transfection. In each well of a 6 -well plate $\sim 6 \times 10^{5}$ cells were seeded, and $24 \mathrm{~h}$ later, the cells were transfected with 200 pmol miR-125b mimics, inhibitors, mimic NC or inhibitor NC. A vertical horizontal line was scratched with a sterile $200 \mu \mathrm{l}$ pipette tip $6 \mathrm{~h}$ subsequent to transfection. The cells were rinsed with PBS to remove the floating cells. The images of the scratch were captured using a digital camera system and a Leica DMIRB inverted fluorescence microscope (Leica Microsystems GmbH, Wetzlar, Germany), at 0 and $24 \mathrm{~h}$ (magnification, x100). The experiments were performed in triplicate and repeated at least three times.

Transwell assay. A Transwell assay was performed to assess the migratory and invasive ability of cells following transfection. In the assay, Transwell chamber inserts (BD Biosciences, Franklin Lakes, USA) with (for invasion) or without (for migration) Matrigel (BD Biosciences) were used. Cells were transfected with miR-125b mimics, inhibitors, mimic NC or inhibitor NC. A total of $6 \mathrm{~h}$ following the transfection, $1 \times 10^{4}$ transfected cells in $200 \mu \mathrm{l}$ serum-free DMEM medium were seeded in the upper chamber of the insert. The bottoms of the inserts were incubated in the medium with $10 \%$ FBS. Following $36 \mathrm{~h}$ (for migration) or $48 \mathrm{~h}$ (for invasion), cells on the upper membrane were removed using a cotton swab, and those that had migrated or invaded to the bottom of the inserts were stained with crystal violet $(1 \mathrm{mg} / \mathrm{ml}$; Sigma-Aldrich; Merck Millipore) for $15 \mathrm{~min}$ at room temperature and counted using a Leica DMIRB inverted microscope (magnification, $\mathrm{x} 200$ ). The experiments were performed in triplicate and repeated at least three times.

Flow cytometry assay. The apoptotic rate of cells was determined by performing a flow cytometry assay with Dead Cell Apoptosis kit with Annexin V fluorescein isothiocyanate (FITC) and propidium iodide (PI) (all from Invitrogen; Thermo Fisher Scientific, Inc.). In each well of a 6-well plate, $\sim 3 \times 10^{5} 786-\mathrm{O}$ or ACHN cells were seeded, and $24 \mathrm{~h}$ later, cells were transfected with miR-125b mimics, inhibitor, mimic NC or inhibitor NC. At $48 \mathrm{~h}$ post-transfection, all cells were harvested and washed twice with cold PBS. Following re-suspension in $100 \mu \mathrm{l}$ of binding buffer, cells were stained with $5 \mu \mathrm{l}$ Annexin V-FITC and $3 \mu \mathrm{l}$ PI. Following $15 \mathrm{~min}$, $400 \mu \mathrm{l}$ of binding buffer was added to each tube. Flow cytometry (EPICS, Xl-4; Beckman Coulter, Inc., Brea, CA, USA) was then used to evaluate the apoptosis rate. The experiments were performed in triplicate and repeated at least three times.

Statistical analysis. The paired $t$-test was used to compare the expression levels of miR-125b in matched tissues. Student's $t$-test was used to analyze assays for characterizing phenotypes of cells. All statistical analyses were performed by 


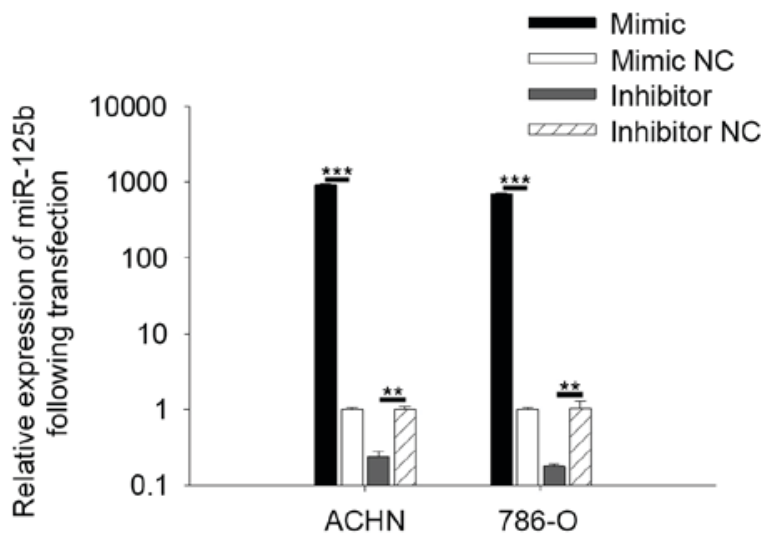

Figure 2. Expression levels of miR-125b in 786-O and ACHN cells following transfection of miR-125b mimic, inhibitor, mimic NC or inhibitor NC. ${ }^{* * *} \mathrm{P}<0.01,{ }^{* * * *} \mathrm{P}<0.001$. NC, negative control; miR, microRNA.

SPSS 19.0 (SPSS, Inc., Chicago, IL, USA). P<0.05 was considered to indicate a statistically significant difference.

\section{Results}

miR-125b was upregulated in renal cell carcinoma (RCC) tissues compared with normal tissues. The expression of miR-125b in tissues was detected by qPCR. The ratios of miR-125b expression in paired tissues [ $\log _{2}$ Ratio tumor tissues (T)/normal adjacent tissues $(\mathrm{N})]$ are presented in Fig. 1A, in which miR-125b was upregulated in 17 RCC tissues. The results of qPCR also demonstrated that the expression level of miR-125b (with mean relative expression 9.72) in RCC tissues was significantly higher compared with in normal tissues $(\mathrm{P}<0.01)$, as demonstrated in Fig. 1B.

Validation of cell transfection efficiency. To quantify the expression of miR-125b in RCC cells following transfection, qPCR was performed. The results indicated that expression of miR-125b in cells transfected with miR-125b mimic was significantly 704.36 (786-O) and 926.44 (ACHN) times higher compared with cells transfected with mimic NC $(\mathrm{P}<0.001)$. Expression of miR-125b in cells transfected with miR-125b inhibitor was 0.18 (786-O) and 0.24 (ACHN) times higher than the cells transfected with inhibitor $\mathrm{NC}(\mathrm{P}<0.01$; Fig. 2).

miR-125b has no marked effect on the proliferation of renal cell carcinoma (RCC) cells. MTT and CCK-8 assays were performed to detect the proliferation of cells. The two assays revealed that overexpression or downregulation of miR-125b had no effect on RCC 786-O and ACHN cell proliferation. The OD values of cells transfected with miR-125b mimic were not significantly different from those of cells transfected with mimic NC ( $>0.05)$, and no significant difference was observed between the inhibitor and inhibitor NC group $(\mathrm{P}>0.05)$. The results are presented in Fig. 3 .

miR-125b promoted cell mobility. Transwell and cell scratch assays were performed to assess the impact of miR-125b on cell mobility. The results of the cell scratch assay are demonstrated in Fig. 4. As presented in Fig. 4A, overexpression of miR-125b promoted the 786-O cell migratory distances by $53.93 \%(\mathrm{P}<0.01)$, while downregulation of miR-125b
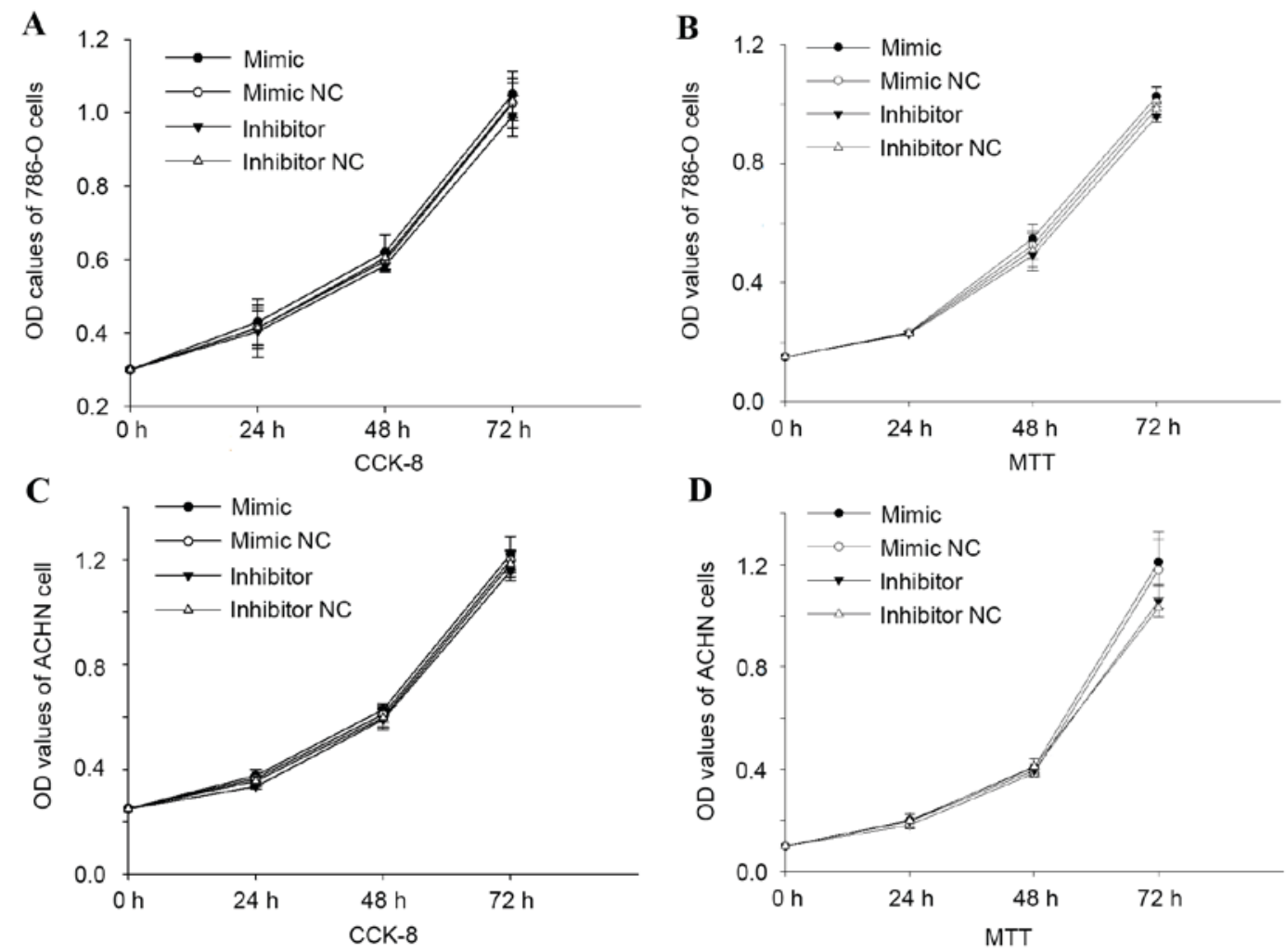

Figure 3. Cell proliferation assay of ACHN and 786-O cells. The results of (A) CCK-8 and (B) MTT assays for 786-O cells, and (C) CCK-8 and (D) MTT assays for ACHN cells demonstrated that miR-125b had no effect on cell proliferation. CCK-8, Cell Counting Kit-8; NC, negative control; OD, optical density. 


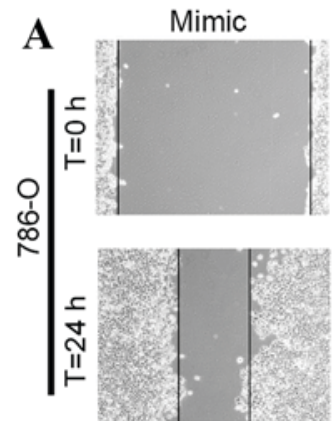

B

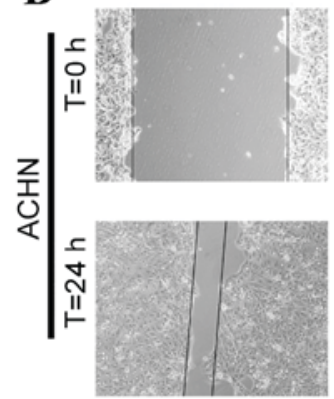

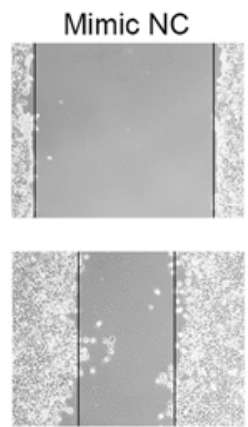
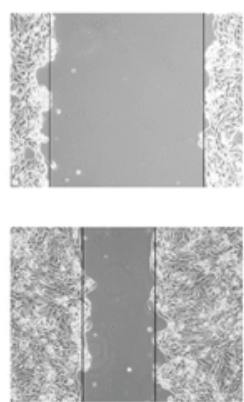
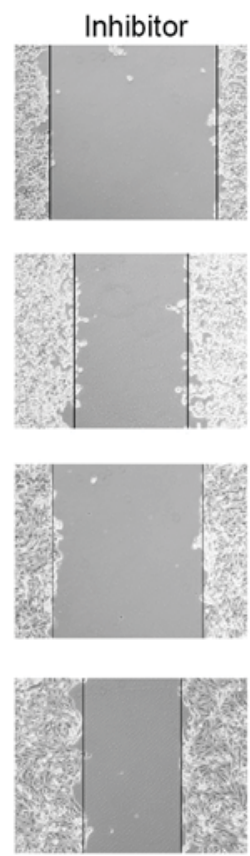

Inhibitor NC
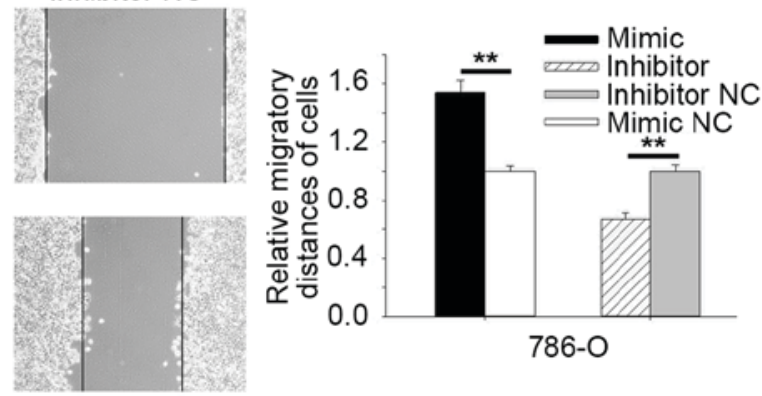

$786-0$

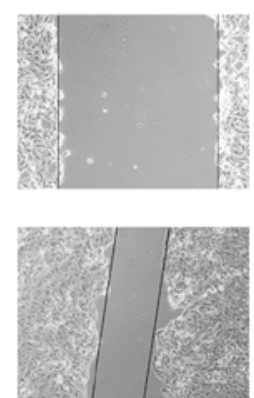

Figure 4. (A) Cell scratch assay of 786-O cells demonstrated that overexpression of miR-125b promoted cell migration. (B) Cell scratch assay of ACHN cells demonstrated that downregulation of miR-125b inhibited cell migration. ${ }^{* *} \mathrm{P}<0.01$. NC, negative control; $\mathrm{T}$, tumor.
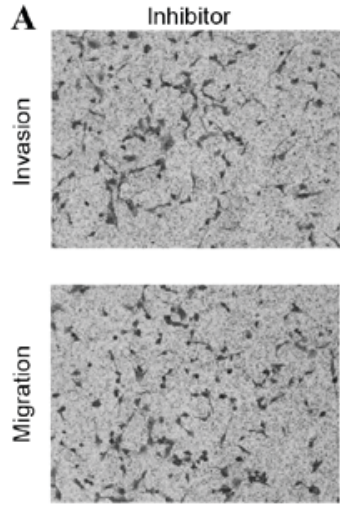

C

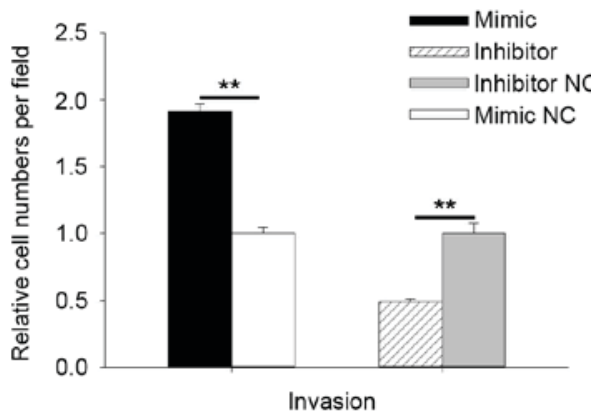

B
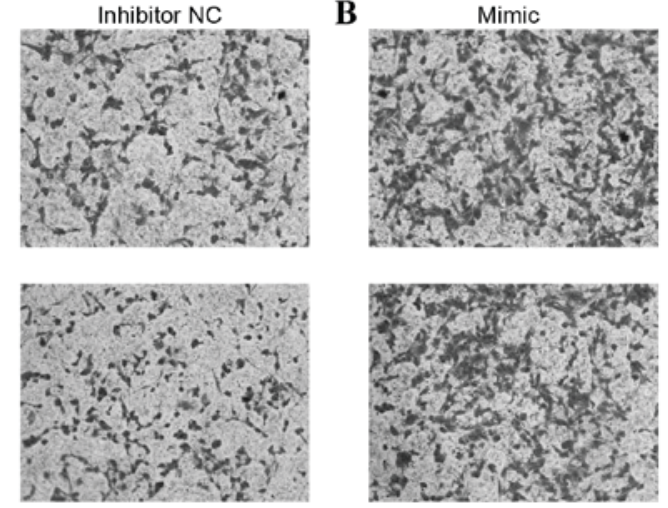

D

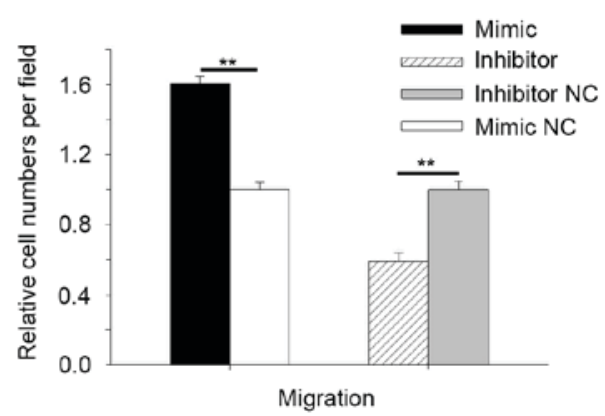

Figure 5. MicroRNA-125b could regulate 786-O cell invasion and migration, as shown by representative images of (A) invasion and (B) migration assays, and quantifications of the $(\mathrm{C})$ invasion and (D) migration assays. ${ }^{* *} \mathrm{P}<0.01$. NC, negative control.

reduced the migratory distances by $33.25 \%(\mathrm{P}<0.01)$. In ACHN cells, overexpression of miR-125b promoted migratory distances by $62.59 \%(\mathrm{P}<0.01)$, and downregulation of miR125b reduced cell migratory distances by $51.99 \%$ $(\mathrm{P}<0.01$; Fig. 4B).

The results of the Transwell assay for 786-O cells are shown in Fig. 5. In Fig. 5A, results of the Transwell invasive assay revealed that upregulation of miR-125b promoted cell invasive ability by $91.71 \%(\mathrm{P}<0.01)$ and downregulation of miR-125b reduced cell invasive ability by $51.43 \%(\mathrm{P}<0.01)$. For the Transwell migratory assay, the migratory cells that were transfected with miR-125b mimic were 1.60 times higher compared with cells transfected with mimic NC $(\mathrm{P}<0.01)$. Migratory cells transfected with miR-125b inhibitor 
A
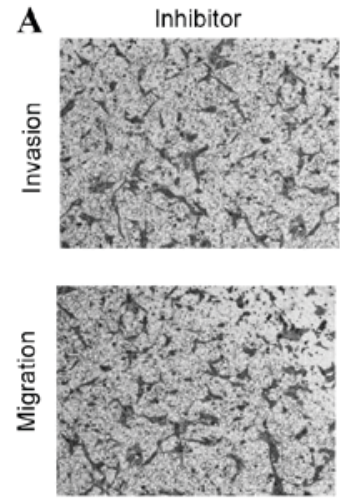
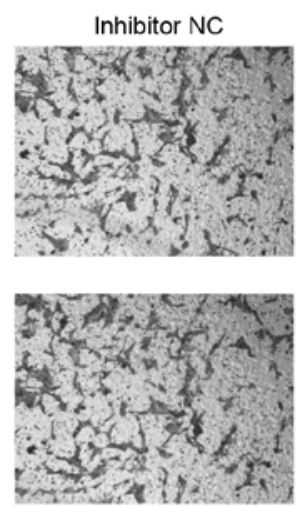

C

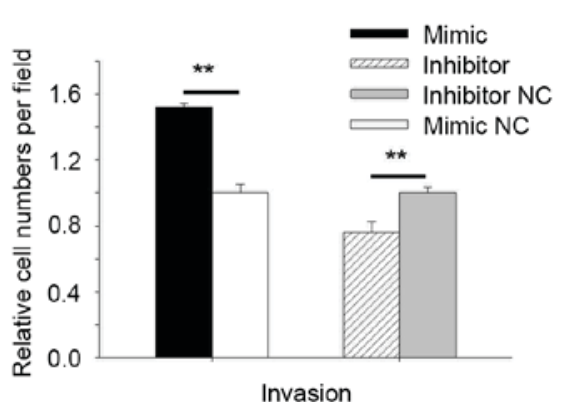

B
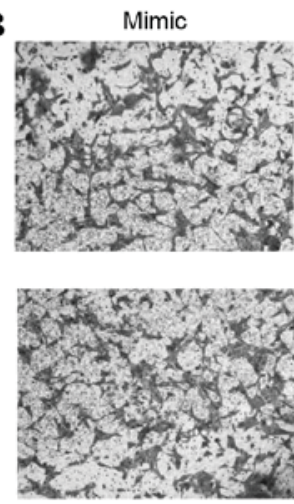
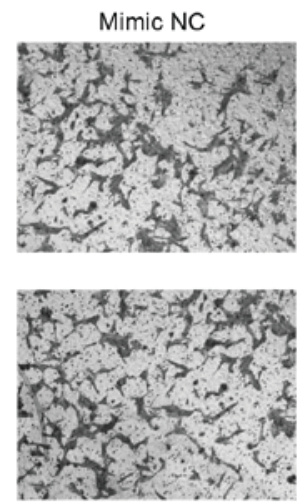

D

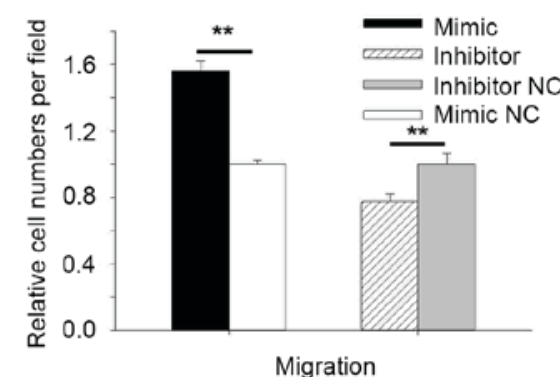

Figure 6. MicroRNA-125b may regulate ACHN cell invasion and migration as demonstrated by representative images of (A) invasion and (B) migration assays, and quantifications of the (C) invasion and (D) migration assays. ${ }^{* *} \mathrm{P}<0.01$. NC, negative control.
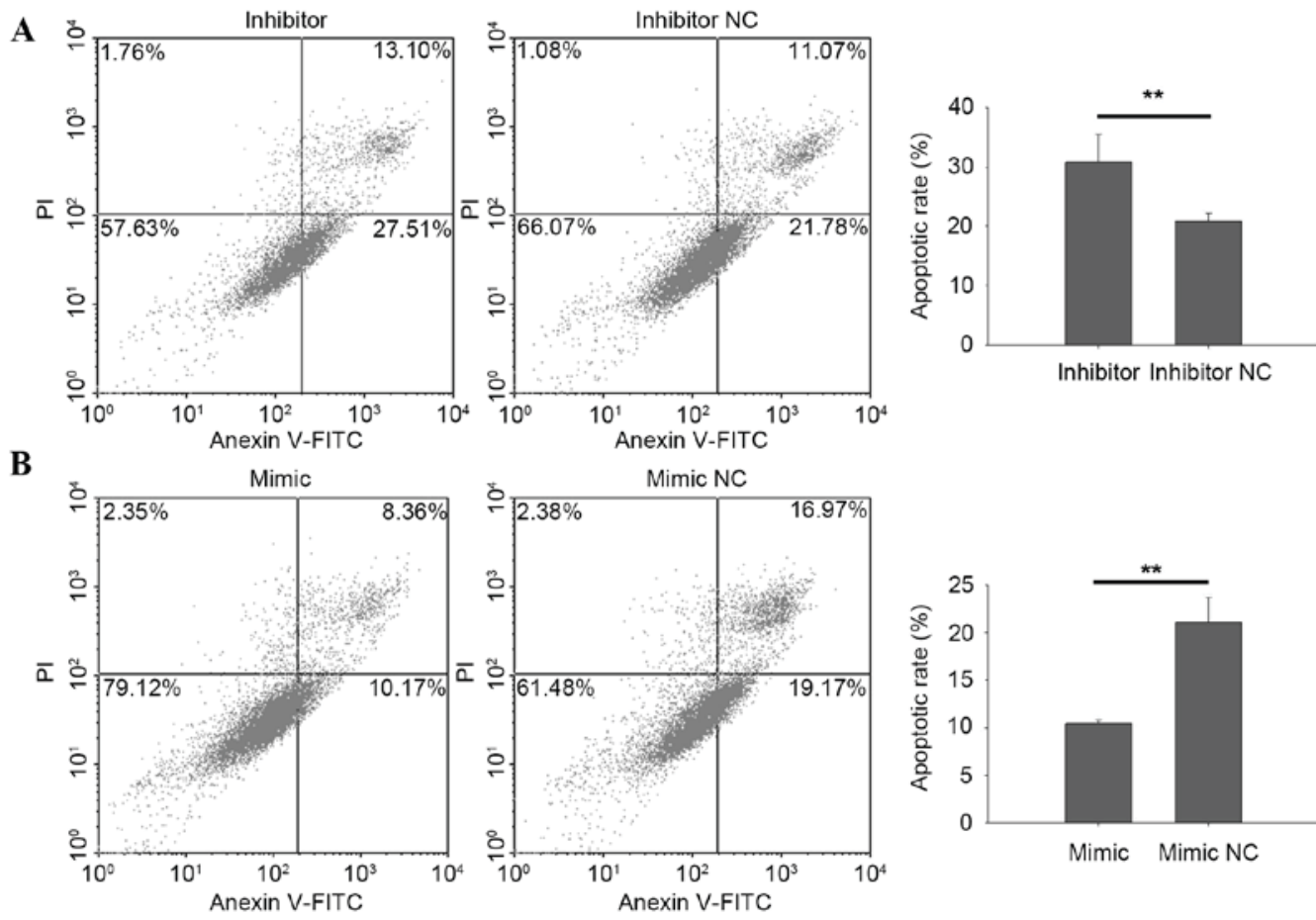

Figure 7. Results of flow cytometry in 786-O cells. (A) Downregulation of miR-125b induced cell apoptosis. (B) Overexpression of miR-125b reduced cell apoptosis. ${ }^{* *} \mathrm{P}<0.01$. PI, propidium iodide; FITC, fluorescein isothiocyanate; $\mathrm{NC}$, negative control.

were 0.59 times higher compared with cells transfected with inhibitor NC ( $\mathrm{P}<0.01$; Fig. 5B). In ACHN cells, the Transwell invasion assay (Fig. 6A) showed that invasive ability of cells transfected with miR-125b mimic was promoted by $52.07 \%$ $(\mathrm{P}<0.01)$ and reduced by $23.88 \%(\mathrm{P}<0.01)$ for cells transfected with inhibitor compared with mimic NC or inhibitor NC. The migratory ability of cells was promoted by $56.21 \%(\mathrm{P}<0.01)$ in the mimic group and reduced by $22.66 \%(\mathrm{P}<0.01)$ in the inhibitor group, compared with the mimic $\mathrm{NC}$ or inhibitor NC group (Fig. 6B). The results of Transwell and cell scratch assays revealed that miR-125b is associated with $\mathrm{RCC}$ cell mobility. 

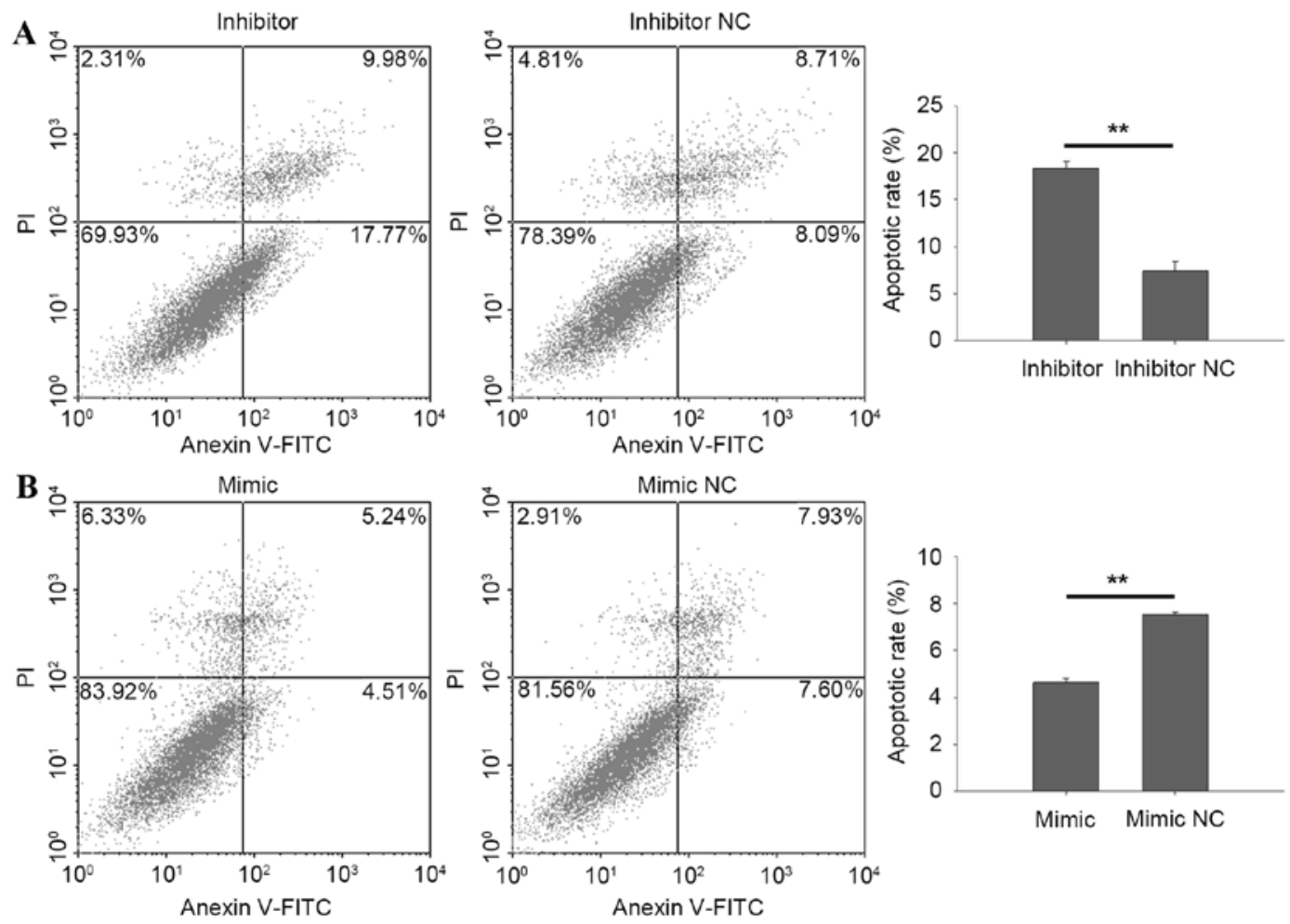

Figure 8. Results of flow cytometry in ACHN cells. (A) Downregulation of miR-125b induced cell apoptosis. (B) Overexpression of miR-125b reduced cell apoptosis. ${ }^{* *} \mathrm{P}<0.01$. PI, propidium iodide; FITC, fluorescein isothiocyanate; $\mathrm{NC}$, negative control.

miR $-125 b$ regulates cell apoptosis. Flow cytometry was performed to detect the apoptosis rate of cells following transfection. As presented in Fig. 7, the apoptotic rate of 786-O cells transfected with miR-125b inhibitor or inhibitor NC was $30.85 \%$ vs. $20.87 \%$ ( $\mathrm{P}<0.01$; Fig. $7 \mathrm{~A}$ ). The apoptotic rate of cells transfected with miR-125b mimic or mimic NC was 10.45 vs. 21.04 ( $\mathrm{P}<0.01$; Fig. 7B). The apoptotic rate of ACHN cells transfected with miR-125b inhibitor was $18.30 \%$ with $7.39 \%$ for cells transfected with inhibitor NC $(\mathrm{P}<0.01$; Fig. 8A). The apoptotic rate of cells transfected with miR-125b mimic or mimic NC was 4.64 vs. $7.52(\mathrm{P}<0.01$; Fig. 8B). The results showed that miR-125b regulates $\mathrm{RCC}$ cell apoptosis.

\section{Discussion}

Deregulation of miRNAs has been detected in numerous types of cancer, including RCC and gastric cancer $(11,16)$, and has been confirmed to be capable of promoting cancer initiation and progression (11), a number of which function as oncogenes or tumor suppressors, depending on the differences of the targeted gene. The expression of miR-125b has been revealed to be deregulated in a number of types of cancer, including gastric cancer (16), non-small-cell lung cancer (NSCLC) (19) and osteosarcoma (12). Numerous previous studies about gene microarray in $\mathrm{RCC}$ revealed that miR-125b was downregulated in ccRCC tissues (20-22). However, another study identified that miR-125b was upregulated in ccRCC by performing ISH (17). In the present study, the expression of miR-125b in RCC tissues and paired adjacent normal tissues was detected and the results revealed that miR-125b was upregulated in RCC tissues compared with adjacent normal tissues. Further study of miR-125b indicated that overexpression of miR-125b could promote cell migration, invasion and reduce cell apoptosis, while downregulation of miR-125b inhibited cell migration, invasion and induced cell apoptosis. The results of CCK-8 and MTT assays showed that miR-125b had no effect on cell proliferation. It can be inferred that the pathway of miR-125b in RCC is associated with cell mobility and apoptosis.

Another study of miR-125b in ccRCC revealed that miR-125b is under-expressed in metastatic tumors, based on the microarray results of 18 patients (23). However, Fu et al (17) demonstrated that miR-125b was upregulated in ccRCC based on 276 samples by ISH, and higher expression of miR-125b predicted shorter survival time and a higher rate of recurrence. The two studies about miR-125b in ccRCC, including the present study, are limited, since the cases were collected from a single institute or area, which could not exclude the bias of area.

A number of studies about miR-125b indicated that miR-125b acted as a tumor suppressor in osteosarcoma, ovarian cancer, hepatocellular carcinoma (HCC) and gastric cancer (15,24-26). In ovarian cancer, miR-125a and miR-125b could inhibit ovarian cancer cell migration, invasion and proliferation by regulating EIF4E-binding protein 1 (24). A study on HCC revealed that the tumor suppressive miR-125b could negatively regulate the expression of long non-coding RNA HOTTIP (25), and low expression of miR-125b was a prognostic marker for poor disease status and outcome (13). It was also revealed that miR-125b could suppress gastric cancer proliferation and invasion by targeting MCL1, and the low expression of miR-125b was associated with advanced clinical stage, lymph node metastases and poor clinical outcomes. 
Thus, miR-125b may be used as a biomarker for gastric cancer (26). Wang et al (15) revealed that tumor suppressive miR-125b could negatively regulate osteosarcoma cell proliferation, invasion and migration, and that overexpression of miR-125b enhanced the chemosensitivity of osteosarcoma cells to cisplatin by targeting Bcl-2. Another study revealed that low expression of miR-125b in osteosarcoma predicted a poor prognosis (12). miR-125b was also downregulated in human papillomavirus-16 (HPV-16) E6 positive esophageal cancer tissues (27). miR-125b has the potential to be used as biomarker for diagnosis, targeted therapy or predicting prognosis.

In NSCLC, miR-125b was identified as an oncogene. Li et al (19) revealed overexpression of miR-125b promoted human NSCLC metastasis by targeting TP53INP1. Another study about miR-125b in lung cancer revealed that knockdown of miR-125b could induce apoptosis and G1/S phase arrest and inhibit the invasive ability of lung cancer cells (28). miR-125b could also regulate kinesin light chain-2, which was upregulated in elderly patients with NSCLC, and could predict a poor clinical outcome of elderly patients with NSCLC (29). Studies of miR-125b in breast cancer revealed that miR-125b was decreased in pre-invasive breast cancer (30) and high expression of miR-125b was associated with poor prognosis in breast cancer (31). In colorectal cancer (CRC), the expression of miR-125b was downregulated (32), while in patients with early colorectal neoplasia (including precancerous lesions and early CRCs) the expression of serum miR-125b was also upregulated compared with healthy controls. Therefore, miR-125b may be used as biomarker for early detection of colorectal neoplasia (33). Thus, the expression of miR-125b in different stages of tumors varies, indicating that the role of miR-125b is constantly changing during the tumorigenesis.

miR-125b has also been shown to serve a role in numerous non-tumor diseases. For example, miR-125b was overexpressed in HPV infected cells and patients and was decreased in patients with invasive cervical carcinoma (34). miR-125b could also protect the ethanol-induced apoptosis and embryotoxicity (35). Due to incomplete binding with the 3'-untranslated region of target mRNA, miR-125b may serve various roles in different diseases or processes $(36,37)$. miRNA may regulate different genes and serve different functions.

In conclusion, the results of the present study revealed that miR-125b was upregulated in RCC tissues and regulated $\mathrm{RCC}$ cell migration, invasion and apoptosis, indicating that miR-125b served a significant role in RCC. Furthermore, the pathway of miR-125b would be identified and the study for miR-125b as a biomarker would be performed.

\section{Acknowledgements}

The present study was supported by the National Natural Science Foundation of China (grant no. 81101922), the Guangdong Natural Science Foundation (grant no. 2015A030313889), the Science and Technology Development Fund Project of Shenzhen (grant nos. JCYJ20120616144352139, JCYJ20130402114702124, JCYJ20140415162542975 and JCYJ20150403091443329) and the Fund of Guangdong Key Medical Subject.

\section{References}

1. Tostain J, Li G, Gentil-Perret A and Gigante M: Carbonic anhydrase 9 in clear cell renal cell carcinoma: A marker for diagnosis, prognosis and treatment. Eur J Cancer 46: 3141-3148, 2010.

2. Hadoux J, Vignot S and De La Motte Rouge T: Renal cell carcinoma: Focus on safety and efficacy of temsirolimus. Clin Med Insights Oncol 4: 143-154, 2010.

3. Su Z, Chen D, Zhang E, Li Y, Yu Z, Shi M, Jiang Z, Ni L, Yang S, Gui Y, et al: MicroRNA-509-3p inhibits cancer cell proliferation and migration by targeting the mitogen-activated protein kinase kinase kinase 8 oncogene in renal cell carcinoma. Mol Med Rep 12: 1535-1543, 2015.

4. Alt AL, Boorjian SA, Lohse CM, Costello BA, Leibovich BC and Blute ML: Survival after complete surgical resection of multiple metastases from renal cell carcinoma. Cancer 117: 2873-2882, 2011.

5. Rasmussen F: Metastatic renal cell cancer. Cancer Imaging 13: 374-380, 2013.

6. Zou X, Zhong J, Li J, Su Z, Chen Y, Deng W, Li Y, Lu S, Lin Y, Luo L, et al: miR-362-3p targets nemo-like kinase and functions as a tumor suppressor in renal cancer cells. Mol Med Rep 13: 994-1002, 2016.

7. Jiang JX, Gao S, Pan YZ, Yu C and Sun CY: Overexpression of microRNA-125b sensitizes human hepatocellular carcinoma cells to 5-fluorouracil through inhibition of glycolysis by targeting hexokinase II. Mol Med Rep 10: 995-1002, 2014.

8. Akman HB, Selcuklu SD, Donoghue MT, Akhavantabasi S, Sapmaz A, Spillane C, Yakicier MC and Erson-Bensan AE: ALCAM is indirectly modulated by miR-125b in MCF7 cells. Tumour Biol 36: 3511-3520, 2015.

9. Yang Q, Wang Y, Lu X, Zhao Z, Zhu L, Chen S, Wu Q, Chen C and Wang Z: MiR-125b regulates epithelial-mesenchymal transition via targeting Sema4C in paclitaxel-resistant breast cancer cells. Oncotarget 6: 3268-3279, 2015.

10. Wei X, Chen D, Lv T, Li G and Qu S: Serum microRNA-125b as a potential biomarker for glioma diagnosis. Mol Neurobiol 53: 163-170, 2016.

11. Iwamoto H, Kanda Y, Sejima T, Osaki M, Okada F and Takenaka A: Serum miR-210 as a potential biomarker of early clear cell renal cell carcinoma. Int J Oncol 44: 53-58, 2014.

12. Karbasy SH, Taheriazam A, Mirghasemi A, Sedaghati F, Shakeri M, Yahaghi E and Bahador R: RETRACTED ARTICLE: Upregulation of miR-300 and downregulation of miR-125b act as potential predictor biomarkers in progression, metastasis, and poor prognosis of osteosarcoma, Tumour Biol: Sep 2, 2015 (Epub ahead of print).

13. Tsang FH, Au V, Lu WJ, Shek FH, Liu AM, Luk JM, Fan ST, Poon RT and Lee NP: Prognostic marker microRNA-125b inhibits tumorigenic properties of hepatocellular carcinoma cells via suppressing tumorigenic molecule eIF5A2. Dig Dis Sci 59: 2477-2487, 2014.

14. He H, Xu F, Huang W, Luo SY, Lin YT, Zhang GH, Du Q and Duan RH: miR-125a-5p expression is associated with the age of breast cancer patients. Genet Mol Res 14: 17927-17933, 2015.

15. Wang F, Yu D, Liu Z, Wang R, Xu Y, Cui H and Zhao T: miR-125b functions as a tumor suppressor and enhances chemosensitivity to cisplatin in osteosarcoma. Technol Cancer Res Treat 15: NP105-NP112, 2016.

16. Wu JG, Wang JJ, Jiang X, Lan JP, He XJ, Wang HJ, Ma YY, Xia YJ, Ru GQ, Ma J, et al: MiR-125b promotes cell migration and invasion by targeting PPP1CA-Rb signal pathways in gastric cancer, resulting in a poor prognosis, Gastric Cancer 18: 729-739, 2015.

17. Fu Q, Liu Z, Pan D, Zhang W, Xu L, Zhu Y, Liu H and Xu J: Tumor miR-125b predicts recurrence and survival of patients with clear-cell renal cell carcinoma after surgical resection. Cancer Sci 105: 1427-1434, 2014.

18. Livak KJ and Schmittgen TD: Analysis of relative gene expression data using real-time quantitative PCR and the 2(-Delta Delta C(T)) Method. Methods 25: 402-408, 2001.

19. Li Q, Han Y, Wang C, Shan S, Wang Y, Zhang J and Ren T: MicroRNA-125b promotes tumor metastasis through targeting tumor protein 53 -induced nuclear protein 1 in patients with non-small-cell lung cancer. Cancer Cell Int 15: 84, 2015.

20. Liu H, Brannon AR, Reddy AR, Alexe G, Seiler MW, Arreola A, Oza JH, Yao M, Juan D, Liou LS, et al: Identifying mRNA targets of microRNA dysregulated in cancer: With application to clear cell renal cell carcinoma. BMC Sys Biol 4: 51, 2010. 
21. He H, Wang L, Zhou W, Zhang Z, Wang L, Xu S, Wang D, Dong J, Tang C, Tang H, et al: MicroRNA expression profiling in clear cell renal cell carcinoma: Identification and functional validation of key miRNAs. PLoS One 10: e0125672, 2015.

22. Juan D, Alexe G, Antes T, Liu H, Madabhushi A, Delisi C, Ganesan S, Bhanot G and Liou LS: Identification of a microRNA panel for clear-cell kidney cancer. Urology 75: 835-841, 2010.

23. Heinzelmann J, Henning B, Sanjmyatav J, Posorski N, Steiner T, Wunderlich H, Gajda MR and Junker K: Specific miRNA signatures are associated with metastasis and poor prognosis in clear cell renal cell carcinoma. World J Urol 29: 367-373, 2011.

24. Lee M, Kim EJ and Jeon MJ: MicroRNAs 125a and 125b inhibit ovarian cancer cells through post-transcriptional inactivation of EIF4EBP1. Oncotarget 7: 8726-8742, 2016.

25. Tsang FH, Au SL, Wei L, Fan DN, Lee JM, Wong CC, Ng IO and Wong CM: Long non-coding RNA HOTTIP is frequently up-regulated in hepatocellular carcinoma and is targeted by tumour suppressive miR-125b. Liver Int 35: 1597-1606, 2015.

26. Wu S, Liu F, Xie L, Peng Y, Lv X, Zhu Y, Zhang Z and He X: miR-125b suppresses proliferation and invasion by targeting MCL1 in gastric cancer. Biomed Res Int 2015: 365273, 2015.

27. Zang B, Huang G, Wang X and Zheng S: HPV-16 E6 promotes cell growth of esophageal cancer via downregulation of miR-125b and activation of Wnt/ $\beta$-catenin signaling pathway. Int J Clin Exp Pathol 8: 13687-13694, 2015.

28. Wang X, Zhang Y, Fu Y, Zhang J, Yin L, Pu Y and Liang G: MicroRNA-125b may function as an oncogene in lung cancer cells. Mol Med Rep 11: 3880-3887, 2015.

29. Wang M, Zhu X, Sha Z, Li N, Li D and Chen L: High expression of kinesin light chain-2, a novel target of miR-125b, is associated with poor clinical outcome of elderly non-small-cell lung cancer patients. Br J Cancer 112: 874-882, 2015.

30. Lehmann TP, Korski K, Gryczka R, Ibbs M, Thieleman A, Grodecka-Gazdecka S and Jagodziński PP: Relative levels of let-7a, miR-17, miR-27b, miR-125a, miR-125b and miR-206 as potential molecular markers to evaluate grade, receptor status and molecular type in breast cancer. Mol Med Rep 12: 4692-4702, 2015.
31. Vilquin P, Donini CF, Villedieu M, Grisard E, Corbo L, Bachelot T, Vendrell JA and Cohen PA: MicroRNA-125b upregulation confers aromatase inhibitor resistance and is a novel marker of poor prognosis in breast cancer. Breast Cancer Res 17: 13, 2015.

32. Chen $\mathrm{H}$ and $\mathrm{Xu} \mathrm{Z}$ : Hypermethylation-associated silencing of miR-125a and miR-125b: A potential marker in colorectal cancer. Dis Markers 2015: 345080, 2015

33. Yamada A, Horimatsu T, Okugawa Y, Nishida N, Honjo H, Ida H, Kou T, Kusaka T, Sasaki Y, Yagi M, et al: Serum miR-21, miR-29a, and miR-125b are promising biomarkers for the early detection of colorectal neoplasia. Clin Cancer Res 21: 4234-4242, 2015.

34. Ribeiro J, Marinho-Dias J, Monteiro P, Loureiro J, Baldaque I, Medeiros R and Sousa H: miR-34a and miR-125b expression in HPV infection and cervical cancer development. Biomed Res Int 2015: 304584, 2015.

35. Chen X, Liu J, Feng WK, Wu X and Chen SY: MiR-125b protects against ethanol-induced apoptosis in neural crest cells and mouse embryos by targeting Bak 1 and PUMA. Exp Neurol 271: 104-111, 2015

36. Xie X, Hu Y, Xu L, Fu Y, Tu J, Zhao H, Zhang S, Hong R and $\mathrm{Gu} \mathrm{X}$ : The role of miR-125b-mitochondria-caspase-3 pathway in doxorubicin resistance and therapy in human breast cancer. Tumour Biol 36: 7185-7194, 2015.

37. Morelli E, Leone E, Cantafio ME, Di Martino MT, Amodio N, Biamonte L, Gullàà $\mathrm{A}$, Foresta U, Pitari MR, Botta C, et al: Selective targeting of IRF4 by synthetic microRNA-125b-5p mimics induces anti-multiple myeloma activity in vitro and in vivo. Leukemia 29: 2173-2183, 2015. 